\title{
A BOOLEAN ALGEBRA WITHOUT PROPER AUTOMORPHISMS
}

\author{
BJARNI JóNSSON
}

It is the purpose of this note to show that there exists an infinite Boolean algebra which has no proper automorphisms. ${ }^{1}$ We shall construct a simply ordered set $S$, introduce a topology on this set in the usual manner, the so-called interval topology determined by the ordering relation, ${ }^{2}$ and prove that $S$ is a compact zero-dimensional Hausdorff space and that the only homeomorphism on $S$ onto $S$ is the identity mapping. It is well known ${ }^{3}$ that the group of automorphisms of the set-field $B$ consisting of all open and closed subsets of $S$ is isomorphic to the group of all homeomorphisms on $S$ onto $S$, whence it follows that $\mathbb{B}$ has no proper automorphisms.

Consider a simply ordered set $S$ with at least two elements. By the interval topology on $S$ we mean the topology which has as a subbasis for open sets, the family of all sets $U \subseteq S$ such that either

$$
U=\{x \mid x \in S \text { and } x<a\} \quad \text { or } U=\{x \mid x \in S \text { and } a<x\}
$$

for some $a \in S$. These sets together with all sets of the form

$$
U=\{x \mid x \in S \text { and } a<x<b\}
$$

with $a, b \in S$ constitute a basis for the interval topology on $S$. We shall need the following theorem.

THEOREM 1. If $S$ is a simply ordered set, then the interval topology on $S$ is a Hausdorff topology. In order for $S$ to be compact and zero-dimensional, it is necessary and sufficient that the following conditions be satisfied:

(i) Every subset of $S$ has a least upper bound and a greatest lower bound in $S$.

(ii) Given any elements $a, b \in S$ with $a<b$, there exist elements $x, y \in S$ such that $a \leqq x<y \leqq b$, and such that $x \leqq u \leqq y$ implies that $u=x$ or $u=y$.

The proof of this theorem offers no difficulty and will be omitted. We shall use certain familiar concepts and results pertaining to

Presented to the Society, February 24, 1951; received by the editors November 22, 1950.

1 This is Problem 74 in G. Birkhoff, Lattice theory, Amer. Math. Soc. Colloquium Publications, vol. 25, rev. ed., 1949, p. 162.

2 Cf. G. Birkhoff, loc. cit. p. 60.

3 Cf. M. H. Stone, Applications of the theory of Boolean rings to general topology, Trans. Amer. Math. Soc. vol. 41 (1937) p. 383. 
simply ordered sets. ${ }^{4}$ Suppose $S$ is a simply ordered set, and let $\omega_{\alpha}$ and $\omega_{\beta}$ be regular initial ordinals (Anfangszahlen). An element $x \in S$ is said to have the character $\left(\omega_{\alpha}, \omega_{\beta}^{*}\right)$ if there exist a strictly increasing transfinite sequence $y=\left\langle y_{0}, y_{1}, \cdots, y_{\xi}, \cdots\right\rangle\left(\xi<\omega_{\alpha}\right)$ such that $x$ is the least upper bound of the elements $y_{\xi}$ and a strictly decreasing transfinite sequence $z=\left\langle z_{0}, z_{1}, \cdots, z_{\eta}, \cdots\right\rangle\left(\eta\left\langle\omega_{\beta}\right)\right.$ such that $x$ is the greatest lower bound of the elements $z_{\eta}$. If $x$ is the least upper bound of a strictly increasing transfinite sequence $y$ $=\left\langle y_{0}, y_{1}, \cdots, y_{\xi}, \ldots\right\rangle$ and if the set of all elements $z \in S$ with $x<z$ is either empty or else has a smallest element, then $x$ is said to have the character $\left(\omega_{\alpha}, 0\right)$. The phrase " $x$ has the character $\left(0, \omega_{\beta}^{*}\right)$ " is defined in a similar manner. Finally, $x$ is said to have the character $(0,0)$ if the set of all elements $y \in S$ with $y<x$ is either empty or else has a largest element and if the set of all elements $z \in S$ with $x<z$ is either empty or else has a smallest element.

It is well known that each element $x \in S$ has one and only one character. We now prove the following theorem.

Theorem 2. Suppose $S$ is a simply ordered set, $f$ maps $S$ homeomorphically onto itself (in the interval topology on $S$ ), and $x \in S$. We then have:

(i) If $x$ has the character $\left(\omega_{\alpha}, \omega_{\beta}^{*}\right)$ where $\alpha \neq \beta$, then $f(x)$ has either the character $\left(\omega_{\alpha}, \omega_{\beta}^{*}\right)$ or $\left(\omega_{\beta}, \omega_{\alpha}^{*}\right)$.

(ii) If $x$ has one of the characters $\left(\omega_{\alpha}, \omega_{\alpha}^{*}\right),\left(\omega_{\alpha}, 0\right),\left(0, \omega_{\alpha}^{*}\right)$, then $f(x)$ also has one of these characters.

(iii) If $x$ has the character $(0,0)$, then $f(x)$ also has the character $(0,0)$.

Proof. Part (iii) is trivial since an element $x \in S$ has the character $(0,0)$ if, and only if, $x$ is an isolated point in the interval topology of $S$.

Suppose $\omega_{\alpha}$ is a regular initial ordinal and assume that there exists a strictly increasing transfinite sequence $y=\left\langle y_{0}, y_{1}, \cdots, y_{\xi}, \cdots\right\rangle$ $\left(\xi<\omega_{\alpha}\right)$ such that $x$ is the least upper bound of the elements $y_{\xi}$. Then $x$ belongs to the closure of the set

$$
A=\left\{y_{\xi} \mid \xi<\omega_{\alpha}\right\} \text {. }
$$

Furthermore, if $x$ belongs to the closure of the set $A^{\prime} \subseteq A$, then $A^{\prime}$ must have $\aleph_{\alpha}$ elements. Let

$B=\{y \mid y \in A$ and $f(y)<f(x)\}$ and $C=\{z \mid z \in A$ and $f(x)<f(z)\}$.

4 Cf. F. Hausdorff, Grundzilge der Mengenlehre, Leipzig, 1914, chap. 6. Our definition of the character of an element differs slightly from the one given there. Thus we assign the characters $\left(\omega_{\alpha}, 0\right),\left(0, \omega_{\beta}^{*}\right)$, and $(0,0)$ to elements which there are given the characters $\left(\omega_{\alpha}, 1\right),\left(1, \omega_{\beta}^{*}\right)$, and $(1,1)$ respectively. We also find it convenient to assign characters to the end points of $S$ in case they exist. 
Then $A=B \cup C$, so that $x$ belongs to the closure of either $B$ or $C$. Suppose $x$ belongs to the closure of $B$, then $f(x)$ belongs to the closure of $f(B)$, whence it follows that $x$ is the least upper bound of $f(B)$. Hence there exists a strictly increasing transfinite sequence $y^{\prime}=\left\langle y_{0}^{\prime}, y_{1}^{\prime}, \cdots, y_{\xi}^{\prime}, \cdots\right\rangle\left(\xi<\omega_{\alpha^{\prime}} \leqq \omega_{\alpha}\right)$ with $y_{\xi}^{\prime} \in f(B)$ such that $f(x)$ is the least upper bound of the elements $y_{\xi}^{\prime}$. It follows that $\alpha^{\prime}=\alpha$. Similarly, if $x$ belongs to the closure of $C$, then there exists a strictly decreasing transfinite sequence $z=\left\langle z_{0}^{\prime}, z_{1}^{\prime}, \cdots, z_{\eta}^{\prime}, \cdots\right\rangle\left(\eta<\omega_{\alpha}\right)$ with $z_{\eta}^{\prime} \in f(C)$ such that $f(x)$ is the greatest lower bound of the elements $z_{\eta}^{\prime}$.

From the above discussion we see that if $x$ has the character $\left(\omega_{\alpha}, \omega_{\beta}^{*}\right)$, then $f(x)$ has one of the characters $\left(\omega_{\alpha}, 0\right)$ or $\left(0, \omega_{\alpha}^{*}\right)$ or else $f(x)$ has a character of the form $\left(\omega_{\alpha}, \omega_{\gamma}^{*}\right)$ or $\left(\omega_{\gamma}, \omega_{\alpha}^{*}\right)$. A similar argument shows that $f(x)$ must have one of the characters $\left(\omega_{\beta}, 0\right)$ or $\left(0, \omega_{\beta}^{*}\right)$ or else $f(x)$ has a character of the form $\left(\omega_{\beta}, \omega_{\delta}^{*}\right)$ or $\left(\omega_{\delta}, \omega_{\beta}^{*}\right)$. Since $f(x)$ has only one character, we infer that if $\alpha \neq \beta$, then $f(x)$ must have either the character $\left(\omega, \omega_{\beta}^{*}\right)$ or $\left(\omega_{\beta}, \omega_{\alpha}^{*}\right)$. Thus (ii) holds.

Now suppose $x$ has one of the characters $\left(\omega_{\alpha}, \omega_{\alpha}^{*}\right),\left(\omega_{\alpha}, 0\right),\left(0, \omega_{\alpha}^{*}\right)$. Then $f(x)$ has either one of these three characters or else $f(x)$ has a character of the form $\left(\omega_{\alpha}, \omega_{\gamma}^{*}\right)$ or $\left(\omega_{\gamma}, \omega_{\alpha}^{*}\right)$ with $\gamma \neq \alpha$. However, applying (i) with $f$ and $x$ replaced by $f^{-1}$ and $f(x)$, we see that in the latter case $x$ would have one of the characters $\left(\omega_{\alpha}, \omega_{\gamma}^{*}\right),\left(\omega_{\gamma}, \omega_{\alpha}^{*}\right)$. Since this contradicts our assumption, the former case must apply, and (ii) holds. The proof is complete.

THEOREM 3. There exists an infinite compact zero-dimensional topological space $S$ such that the only homeomorphism on $S$ onto $S$ is the identity mapping.

Proof. Let $\kappa_{0}=0$ and $\kappa_{n+1}=\omega_{\kappa_{n}}$ for $n=0,1, \cdots$, and for each $k<\omega_{0}$ let $A_{k}$ be the family of all sequences $x=\left\langle x_{0}, x_{1}, \cdots\right.$, $x_{n}, \cdots>$ of ordinals $x_{n}$ such that $x_{n}<\kappa_{n+1}$ for $n=0,1, \cdots, k$ and $x_{n}=0$ for $n=k+1, k+2, \cdots$. Then $A_{0} \subseteq A_{1} \subseteq \cdots \subseteq A_{n} \subseteq \cdots$, and the cardinal number of $A_{k}$ is $\aleph_{k_{k}}$. The set $B_{k}$ of all ordinals $\alpha$ with $\kappa_{k} \leqq \alpha<\kappa_{k+1}$ also has $\aleph_{\kappa_{k}}$ elements, hence there exists a univalent function $f_{k}$ on $A_{k}$ to $B_{k}$.

We next construct for each $k<\omega_{0}$ a subset $C_{k}$ of $A_{k}$. Let $C_{0}=A_{0}$, and, assuming that $C_{k}$ has already been defined, consider a sequence $x \in A_{k+1}$. Then the sequence $x^{\prime}=\left\langle x_{0}, x_{1}, \cdots, x_{k}, 0,0, \cdots\right\rangle$ is a member of $A_{k}$. We let $x \in C_{k+1}$ if and only if $x^{\prime} \in C_{k}$ and $x_{k+1}<\omega_{\alpha}$ where $\alpha=f_{k}\left(x^{\prime}\right)+1$. Thus $C_{k}$ is defined for each $k<\omega_{0}$; we denote the settheoretical union of all the sets $C_{k}$ by $C$.

Let $\leqq$ be the lexicographic ordering of $C$; that is, given two se- 
quences $x, y \in C$, we let $x \leqq y$ if, and only if, $x=y$ or else $x_{k}<y_{k}$ where $k$ is the smallest natural number such that $x_{k} \neq y_{k}$. This relation simply orders $C$ and well orders each of the sets $C_{k}$.

Let $D_{0}$ be the set of all ordered pairs $\langle x, 0\rangle$ with $x \in C$ such that $x \neq\langle 0,0,0, \cdots\rangle$ and the last nonzero term of $x$ is not a limiting ordinal, let $D_{1}$ be the set of all ordered pairs $\langle x, 1\rangle$ with $x \in C$, and let $D=D_{0} \cup D_{1}$. For two ordered pairs $\langle x, i\rangle,\langle y, j\rangle$ in $D$ we write $\langle x, i\rangle \leqq\langle y, j\rangle$ if, and only if, either $x<y$ or else $x=y$ and $i \leqq j$.

The simply ordered set $D$ can be imbedded in a simply ordered set $S$ such that every subset of $S$ has a least upper bound and a greatest lower bound in $S$, and such that each element $\mathfrak{p} \in S$ is the least upper bound of all elements $q \in D$ with $q \leqq p$ and the greatest lower bound of all elements $\mathfrak{r} \in D$ with $\mathfrak{p} \leqq \mathfrak{r}$. We shall prove that the interval topology on $S$ satisfies the conditions of the theorem.

The set $C$ is dense in itself. In fact, suppose $x, y \in C$ and $x<y$. If $n$ is the smallest natural number such that $x_{n} \neq y_{n}$, then $x_{n}<y_{n}$, the sequence $z=\left\langle x_{0}, x_{1}, \cdots, x_{n}, x_{n+1}+1,0,0, \cdots\right\rangle$ is a member of $C$, and $x<z<y$.

Suppose $\mathfrak{p}, \mathfrak{q} \in S$ and $\mathfrak{p}<\mathfrak{q}$. Then $\mathfrak{p} \leqq \mathfrak{r}<\boldsymbol{s} \leqq \mathfrak{q}$ for some $\mathfrak{r}, \mathfrak{s} \in D$, and we have $\mathfrak{r}=\langle x, i\rangle$ and $\varepsilon=\langle y, j\rangle$ with $x, y \in C$ and $i, j=0,1$. If $x=y$, then $i=0$ and $j=1$, and there exists no element $\mathrm{t} \in S$ with $\mathfrak{r}<\mathrm{t}<8$. If $x<y$, then $x<z<y$ for some $z \in C$. First suppose the last nonzero term $z_{n}$ of $z$ is a limiting ordinal. Then $z$ is the least upper bound of the sequences $\left\langle z_{0}, z_{1}, \cdots, z_{n-1}, \alpha+1,0,0, \cdots\right\rangle$ with $\alpha<z_{n}$. Hence one of these sequences must be larger than $x$. We therefore see that $z$ can be so chosen that the last nonzero term is not a limiting ordinal. Letting $\mathfrak{r}^{\prime}=\langle z, 0\rangle$ and $\mathfrak{s}^{\prime}=\langle z, 1\rangle$, we thus have $\mathfrak{r}^{\prime}, \boldsymbol{z}^{\prime} \in D \subseteq S$ and $\mathfrak{p} \leqq \mathfrak{r}^{\prime}<\mathcal{B}^{\prime} \leqq \mathfrak{q}$, and there exists no element $\mathfrak{t} \in S$ with $\mathfrak{r}^{\prime}<\mathfrak{t}<\mathcal{B}^{\prime}$. We conclude by Theorem 1 that $S$ is compact and zero-dimensional.

From the discussion in the preceding paragraph it is clear that the set $D_{0}$ is everywhere dense in $S$ in the interval topology. In order to prove that the only homeomorphism on $S$ onto $S$ is the identity mapping, it is therefore sufficient to show that every homeomorphism on $S$ onto $S$ maps each member of $D_{0}$ onto itself. For this purpose we study the characters of the elements of $S$.

Suppose $\mathfrak{p} \in D_{0}$. Then $\mathfrak{p}=\langle x, 0\rangle$ where $x \in C$ and the last nonzero term $x_{n}$ of $x$ is not a limiting ordinal. Hence $x_{n}=u+1$ for some ordinal $u$. The sequence $x^{\prime}=\left\langle x_{0}, x_{1}, \cdots, x_{n-1}, u, 0,0, \cdots\right\rangle$ is then a member of $C$. Letting $\alpha=f_{n}\left(x^{\prime}\right)+1$ and $y^{\beta}=\left\langle x_{0}, x_{1}, \cdots, x_{n-1}\right.$, $u, \beta, 0,0,0, \cdots\rangle$ for every $\beta<\omega_{\alpha}$, we see that all the sequences $y^{\beta}$ are members of $C$, and that their least upper bound is $x$. Hence $\mathfrak{p}$ is the least upper bound of all the ordered pairs $\left\langle y^{\beta}, 1\right\rangle$ with $\beta<\omega_{\alpha}$, and 
we conclude that $\mathfrak{p}$ has the character $\left(\omega_{\alpha}, 0\right)$. Recalling how the functions $f_{n}$ were chosen, we see that distinct members of $D_{0}$ have different characters.

Suppose $\mathfrak{p} \in D_{1}$. Then $\mathfrak{p}=\langle x, 1\rangle$ with $x \in C$. Choosing $n$ sufficiently large we have $x_{n}=x_{n+1}=\cdots=0$. For each $k<\omega_{0}$ we let $z^{k}$ $=\left\langle z_{0}^{k}, z_{1}^{k}, \cdots, z_{m}^{k}, \cdots\right\rangle$ where $z_{m}^{k}=x_{m}$ for $m<n, z_{n+k}^{k}=1$, and $z_{m}^{k}=0$ for $n \leqq m \neq n+k$. Then $z^{0}>z_{1}>\cdots>z^{k} \cdots$, and the greatest lower bound of the sequences $z^{k}$ is $x$. Hence the greatest lower bound of the ordered pairs $\left\langle z^{k}, 1\right\rangle$ is $\mathfrak{p}$. We thus see that $\mathfrak{p}$ has either the character $\left(0, \omega_{0}^{*}\right)$ or else a character of the form $\left(\omega_{\alpha}, \omega_{0}^{*}\right)$.

Suppose $p \in S-D$. First assume that $p$ is the largest element of $S$. Observe that the sequences $y^{k}=\langle k, 0,0, \cdots\rangle$ with $k<\omega_{0}$ have no upper bound in $C$. Therefore the least upper bound of the ordered pairs $\left\langle y^{k}, 1\right\rangle$ is $\mathfrak{p}$, whence it follows that the character of $\mathfrak{p}$ is $\left(\omega_{0}, 0\right)$. Next suppose $\mathfrak{p}$ is not the largest element of $S$. Then the set $E$ of all elements $q \in D_{1}$ with $\mathfrak{p}<q$ is nonempty. In fact, if we let $E_{k}$ be the set of all ordered pairs $q=\langle x, 1\rangle$ with $x \in C_{k}$, then $E \cap E_{0}$ is nonempty and hence each of the sets $E \cap E_{k}$ is nonempty. Since $E_{k}$ is well ordered, each of the sets $E \cap E_{k}$ has a smallest element $q^{k}$. Hence $\mathfrak{q}^{0} \geqq q^{1} \geqq \cdots \geqq \mathfrak{q}^{k} \geqq \cdots$, and the greatest lower bound of the elements $\mathfrak{q}^{k}$ is $\mathfrak{p}$. Hence $\mathfrak{p}$ has a character of the form $\left(\omega_{\alpha}, \omega_{0}^{*}\right)$.

Suppose $\phi$ is a homeomorphism on $S$ onto $S$, and consider an element $p \in D_{0}$. Then $p$ has a character of the form $\left(\omega_{\alpha}, 0\right)$ with $\alpha>0$, whence it follows by Theorem 2 that $\phi(p)$ has one of the characters $\left(\omega_{\alpha}, 0\right),\left(0, \omega_{\alpha}^{*}\right),\left(\omega_{\alpha}, \omega_{\alpha}^{*}\right)$. But no other member of $S$ has one of these characters, so that we must have $\phi(p)=p$. Since this holds for every $\mathfrak{p} \in D_{0}$, we conclude that $\phi$ is the identity mapping. The proof is complete.

We now obtain the following theorem.

Theorem 4. There exists an infinite Boolean algebra which has no proper automorphisms.

PROOF. This is proved by Theorem 3 and the introductory remarks.

BROWN UNIVERSITY 